\title{
FREE ABELIAN TOPOLOGICAL GROUPS AND THE PONTRYAGIN-VAN KAMPEN DUALITY
}

\author{
Vladimir Pestov
}

\begin{abstract}
We study the class of Tychonoff topological spaces such that the free Abelian topological group $A(X)$ is reflexive (satisfies the Pontryagin-van Kampen duality). Every such $X$ must be totally path-disconnected and (if it is pseudocompact) must have a trivial first cohomotopy group $\pi^{1}(X)$. If $X$ is a strongly zero-dimensional space which is either metrisable or compact, then $A(X)$ is reflexive.
\end{abstract}

\section{INTRODUCTION}

In this paper we investigate to what extent the machinery of the Pontryaginvan Kampen duality is applicable to the study of Markov free topological groups [14] on completely regular topological spaces. A topological group is called reflexive if it is canonically isomorphic to the second character group, and the Pontryagin-van Kampen duality provides a fine tool for analysing the structure of such groups. Among the best known classes of reflexive groups are locally compact Abelian groups, their direct products [12], and additive groups of Banach spaces [27]. However, free Abelian topological groups are not to be found in any of these classes - unless $X$ is discrete, $A(X)$ is neither locally compact nor metrisable (see, for example, $[1,4.11$ and 4.14]). Therefore, the problem of singling out those spaces $X$ for which the group $A(X)$ is reflexive is of some interest.

It is known that for a vast class of spaces $X$ the group $A(X)$ is non-reflexive this is the case if at least one path component of a $k_{\omega}$-space $X$ contains more than one point. (A $k_{\omega}$-space, $X$, is a union of countably many compact subspaces carrying the weak topology with respect to this family.) This result of a negative character, due to Nickolas [18], remained for some years the only known bit of information about the reflexivity of the groups $A(X)$.

In the present paper the character group $A(X)^{\wedge}$ is computed and certain necessary or sufficient conditions for the group $A(X)$ to be reflexive are analysed. In particular, it is shown that total path-disconnectedness forms such a necessary condition for every space $X$. For the first time the results in a positive direction are obtained: it is shown in Section 5 that $A(X)$ is reflexive for a rather wide class of spaces $X$, containing,

Received 6th December 1994.

Copyright Clearance Centre, Inc. Serial-fee code: 0004-9729/95 \$A2.00+0.00. 
in particular, all zero-dimensional compact spaces and all strongly zero-dimensional metrisable spaces.

The present paper was originially written (in Russian) in early 1983, while the author was a $\mathrm{PhD}$ student at Moscow State University, and this research had been stimulated by Morris's book [16] (which had just become available in Russian translation) and Noble's paper [19]; the author's thanks go to his $\mathrm{PhD}$ advisor, Prof. A.V. Arhangel'skiir, for bringing Noble's work to his attention. The results of the paper were not included in the author's $1983 \mathrm{PhD}$ thesis, and only a fraction of them, practically without proofs, were announced in a minuscule 1985 note [22] in a rather obscure journal, not readily available beyond (ex)USSR's borders. For a number of years that followed the author deemed the manuscript lost.

The decision to publish the results now, more than a decade later, was motivated by an unceasing, yet moderate, flow of research on Pontryagin-van Kampen duality (see [3] or the recent elegant notes [27] and [15]). In general terms, dual objects, of which the character groups form a simple commutative speciman, are of paramount importance in quantum group theory and noncommutative analysis and geometry; on the other hand, various close relatives of free Abelian topological groups seem to gain in significance as well (see our recent survey [23]).

\section{Preliminaries}

All topological spaces are assumed to be completely regular $T_{1}$-spaces. For a topological group $G$ we denote by $G^{\wedge}$ the group of characters of $G$ (continuous homomorphisms to the circle group $\mathbb{T}$ ). A subset $A$ of a topological group $G$ is precompact if it can be covered by finitely many translations of an arbitrary neighbourhood of the identity of $G$. For our purpose it is more convenient, following Raikov [24], to equip $G^{\wedge}$ with the topology of uniform convergence on all precompact subsets of $G$ (instead of the ordinary compact-open topology; however, we shall observe soon that for a very broad class of topological groups the two resulting dualities are identical.) By $\nu: G \rightarrow G^{\wedge \wedge}$ we denote the canonical homomorphism from $G$ to the second character group: $\nu(g)(\chi)=\chi(g)$ for all $g \in G$ and $\chi \in G^{\wedge}$. A group $G$ is called reflexive if $\nu$ is a topological isomorphism onto.

By $\widehat{G}$ we denote the completion of a Hausdorff topological group (with respect to the two-sided uniformity, see $[\mathbf{2 5}, \mathbf{2 0}])$.

An arbitrary space $X$ sits as a closed topological subspace in the (Markov) free Abelian topological group $A(X)$ on $X$; the group $A(X)$ is algebraically free on $X$ and every continuous mapping $f: X \rightarrow G$, where $G$ is any Abelian topological group, extends in a unique fashion to a continuous homomorphism $\bar{f}: A(X) \rightarrow G$ (see [14, 10, 1]. A solid survey on the subject is [17]; a number of fine results on the structure of 
$A(X)$ are contained in [29]).

We denote by $L(X)$ the free locally convex space on $X[14,6,7,26,30]$. It contains $X$ as a closed topological subspace and as a vector basis in such a way that every continuous mapping $f: X \rightarrow E$, where $E$ is any locally convex space, extends in a unique way to a continuous linear operator $\bar{f}: L(X) \rightarrow E$. The identity map $\operatorname{Id}_{X}$ gives rise to a continuous group homomorphism $\gamma$ from $A(X)$ to the additive group of $L(X)$; Theorem 3 in [29] states that $\gamma$ is a topological isomorphism of $A(X)$ onto a closed subgroup of $L(X)$.

By $\theta X$ we denote the Dieudonne completion of a topological space $X$, that is, the completion of $X$ with respect to the finest compatible uniform structure $\mathcal{U}_{X}$ [5, 8.5.13].

Recall that a topological space $X$ is strongly zero-dimensional $[5,6.2]$ if every finite cover of $X$ consisting of functionally open sets has a finite disjoint open refinement. (A set is functionally open if its completement is the locus of zeros of a continuous realvalued function on $X$.) If $X$ is normal, "functionally" becomes redundant. A space $X$ is strongly zero-dimensional if and only if $\beta X$ is.

A subset $A$ of a space $X$ is termed bounded in $X$ if the restriction to $A$ of every continuous real-valued function $f$ on $X$ is bounded. By $\mu X$ we denote the smallest subspace of the Stone-Čech compactification $\beta X$, containing $X$ and such that every bounded closed subset of $\mu X$ is compact. We say that $X$ is a $\mu$-space if $X=\mu X$. Always $\mu X \subseteq \theta X$ and, in particular, every Dieudonné complete space is a $\mu$-space. (The above definitions and results can be found in [4].)

A topological group $G$ is called a $k$-group [19] if an arbitrary homomorphism $h$ from $G$ to an arbitrary topological group is continuous whenever the restrictions of $h$ to all compact subspaces of $G$ are continuous. The most important examples of $k$-groups are locally compact groups, metrisable groups, and, more generally, all topological groups which are $k$-spaces. Recall also that a space $X$ is called a $k_{f}$ space [2] if an arbitrary real-valued function on $X$ (equivalently: an arbitrary mapping from $X$ to a completely regular space) is continuous as soon as its restriction to every compact subset of $X$ is continuous.

\section{The GROUP $A(X)^{\wedge}$}

Proposition 1. If $X$ is a $k_{f}$-space, then $A(X)$ is a $k$-group.

Proof: Let $f$ be a homomorphism from $A(X)$ to a topological group $G$, and assume that the restriction of $f$ to any compact subset of $G$ is continuous. In particular, the restriction of the map $\left.f^{\prime} \equiv f\right|_{X}$ to any compact subset of $X$ is continuous; since $X$ is a $k$-space, $f^{\prime}$ is continuous, and so is its unique homomorphic extension to $A(X)$, that is, $f$. 
We say that a topological group $G$ is a $p k$-group if any homomorphism $f$ from $G$ to a topological group is continuous as soon as the restriction of $f$ to every precompact subset of $G$ is continuous. Following [30], we call a topological space $X$ a $b_{f}$-space if an arbitrary real-valued function on $X$ is continuous whenever its restriction to every bounded subset of $X$ is continuous.

Lemma 1. A subset $H$ of a space $X$ is bounded in $X$ if and only if $H$ is precompact in $A(X)$.

Proof: The closure of $X$ in the completion of $A(X)$ coincides with $\theta X$. (See [21]; the proof of the Theorem and all results presented in [21] for free topological groups are valid verbatim for free Abelian topological groups.) Boundedness of $H$ is equivalent to compactness of $\mathrm{cl}_{\theta X} H$ ( $\theta X$ is a $\mu$-space), and precompactness of $H$ in $A(X)$ means exactly that the closure of $H$ in the completion of $A(X)$ (and, in fact, in $\theta X)$ is compact.

Proposition 2. If $X$ is a $b_{f}$-space then $A(X)$ is a pk-group.

Proof: This follows from Lemma 1 and is similar to the proof of Proposition 1 . D

Proposition 3. If $G$ is a $p k$-group then the group $G^{\wedge}$ is complete.

Proof: This follows from the fact that $G^{\wedge}$ in the case where $G$ is a $p k$-group can be thought of as the group of all homomorphisms $G \rightarrow \mathbb{T}$ with continuous restrictions to all precompact subsets of $G$, endowed with the topology of uniform convergence on precompact subsets; but such an Abelian group is obviously complete.

We denote by $G^{*}$ the character group of $G$, endowed with the compact-open topology.

PROPOSITION 4. If $G$ is a complete group, then $G^{\wedge}$ and $G^{*}$ are (canonically) topologically isomorphic.

Proof: In a complete group all closed precompact subsets are compact.

Propositions 3 and 4 imply:

Proposition 5. If $G$ is a complete $k$-group then $G^{\wedge \wedge}$ and $G^{* *}$ are (canonically) topologically isomorphic.

For an $x \in A(X)$ we denote by $\operatorname{supp} x$ the set $\left\{x_{1}, \ldots, x_{n}\right\} \subseteq X$, where $x=$ $\sum_{i=1}^{n} \varepsilon_{i} x_{i}, \varepsilon_{i} \in \mathbb{Z} \backslash\{0\}, x_{i} \neq x_{j}$ for $i \neq j$ is an irreducible representation of $x$ in the alphabet $X$. If $B \subseteq A(X)$, we set $\operatorname{supp} B=\bigcup\{\operatorname{supp} x: x \in B\}$.

Proposition 6. (Arhangel'skii) If $C \subseteq A(X)$ and $C$ is precompact, then $\operatorname{supp} C$ is bounded in $X$.

Proof: This follows by applying Proposition 2 in [2] to the spaces $X$ and $A(X)$, 
respectively, and to the correspondence $f \mapsto \bar{f}$, where $f: X \rightarrow \mathbb{R}$ and $\bar{f}$ is a homomorphism from $A(X)$ to $\mathbb{R}$, viewed as a linear map $C(X) \rightarrow C(A(X))$.

We denote by $r$ the restriction map $\left.f \mapsto f\right|_{X}$ from $A(X)^{\wedge}$ to the group $C_{b}(X, \mathbb{T})$ (the subscript " $b$ " denotes the topology of uniform convergence on bounded subsets). For an $H \subseteq X, F \subseteq G$, and and an open subset $U \subseteq \mathbb{T}$ we set

$$
M(F, U) \stackrel{\text { def }}{=}\left\{\chi \in G^{\wedge}: \chi(F) \subseteq U\right\}
$$

(basic sets for the topology of $G^{\wedge}$ ) and

$$
N(H, U) \stackrel{\text { def }}{=}\{f \in C(X, \mathbb{T}): f(H) \subseteq U\}
$$

(basic sets for the topology of $C_{b}(X, \mathbb{T})$ ).

Proposition 7. The group $A(X)^{\wedge}$ is topologically isomorphic to the group $C_{b}(X, \mathbb{T})$ under the restriction mapping $r$.

Proof: The fact that $r$ is an algebraic isomorphism is obvious. Let $C$ be a precompact subset of $A(X)$. It is well known that for some $n \in \mathbb{N}, C \subseteq A(X)_{n}$, where the last symbol stands for the set of all $x \in A(X)$ having reduced length $\leqslant n$ over $X[1]$. If $U$ is an arbitrary neighbourhood of the identity $e$ in $\mathbb{T}$, choose a symmetric neighbourhood $V$ of $e$ in $\mathbb{T}$ such that $V^{n} \subseteq U$. Proposition 6 implies now that $r^{-1}[M(\operatorname{supp} C, V)] \subseteq N(C, U)$, which yields that $r$ is open. Conversely, if $H$ is a bounded subset of $X$, then $H$ is precompact in $A(X)$ (Lemma 1) and $r^{-1}[M(C, U)]=N(C, U)$; this means that $r$ is continuous.

In what follows we identify the topological groups $A(X)^{\wedge}$ and $C_{b}(X, \mathbb{T})$.

2. The evaluation maP $\nu: A(X) \rightarrow A(X)^{\wedge \wedge}$

The reflexivity of a topological group $G$ is equivalent to the following properties of the evaluation map $\nu: G \rightarrow G^{\wedge \wedge}$.

1. $\nu$ is one-to-one.

2. $\nu$ is continuous.

3. $\nu$ is relatively open. (That is, $\nu$ is open as a map from $G$ onto its image in $G^{\wedge \wedge}$.)

4. $\nu$ is onto.

In this section we shall investigate the first three properties for the evaluation map $\nu: A(X) \rightarrow A(X)^{\wedge \wedge}$. The last property is examined in three separate sections, Section 3 and Section 4 (necessary conditions) and Section 5 (sufficient conditions).

1. Injectivity of $\nu$. 
Proposition 8. For every topological space $X$ the mapping $\nu: A(X) \rightarrow$ $A(X)^{\wedge \wedge}$ is a monomorphism.

Proof: This is just a reformulation of a well-known and rather simple fact: continuous characters separate points in free Abelian topological groups (see, for example, [9]).

2. Continuity of $\nu$.

THEOREM 1. If $G$ is a pk-group then the mapping $\nu: G \rightarrow G^{\wedge \wedge}$ is continuous.

Proof: The proof is modelled on Noble's proof of Theorem 2.3 in [19]. Denote by $t^{\prime}$ the topology on $G$, induced by the embedding

$$
G \ni x \mapsto(x, \nu(x)) \in G \times G^{\wedge \wedge}
$$

It is the smallest topology which (a) is finer than the original topology $t$ on $G$, (b) is translation-invariant, and (c) contains all sets of the form $\bigcap_{x \in K} \chi^{-1}(U)$, where $K$ is an arbitrary precompact subset of $G^{\wedge}$ and $e_{\mathbb{T}} \in U \subseteq \mathbb{T}$ is open. The Ascoli Theorem in a somewhat generalised form (see problem 379 in [13]) implies that any precompact subset $K \subseteq G^{\wedge}$ is equicontinuous with respect to the finest topology $t^{\prime \prime}$ on $G$ inducing the originial topology on every precompact subset of $G$. Since $t^{\prime \prime}$ is clearly translationinvariant, one concludes: $t^{\prime \prime} \subseteq t^{\prime} \subseteq t$. The last observation implies that $t^{\prime}$ is the finest group topology on $G$ inducing the originial topology on every precompact subset of $G$. The property of $G$ being a $p k$-group means exactly that $t$ has the same property. Therefore, $t=t^{\prime}$, and $\nu$ is continuous.

The following is an immediate consequence of Theorem 1 and Proposition 2.

CoRollary 1. If $X$ is a $b_{f}$-space then the map $\nu: A(X) \rightarrow A(X)^{\wedge \wedge}$ is continnous.

3. Relative openness of $\nu$.

Let $F, G, H$ be sets, let $A \subseteq G^{F}, B \subseteq H^{G}$. Set $B \circ A=\{f \circ g: f \in B, g \in A\}$.

Lemma 2. Let $F, G, H$ be topological groups, and let $A \subseteq G^{F}, B \subseteq H^{G}$. If the sets $A$ and $B$ are equicontinuous, then $B \circ A$ is equicontinuous.

Proof: This follows from the fact that for every neighbourhood $U$ of the identity in $B$ the set $(B \circ A)^{-1}(U) \equiv A^{-1}\left(B^{-1}(H)\right)$ is open in $F$.

Theorem 2. For every $X$ the topology of $A(X)$ is the topology of uniform convergence on all equicontinuous subsets of $C(X, \mathbb{T})$.

Proof: Let $U$ be an arbitrary neighbourhood of zero in $A(X)$. We shall find an open neighbourhood $W$ of the identity in $\mathbb{T}$ and an equicontinuous subset $H \subseteq C(X, \mathbb{T})$ such that $\bigcap\left\{\bar{h}^{-1}(W): h \in H\right\} \subseteq U$. 
Let $V$ be a neighbourhood of zero in the free locally convex space $L(X)$ such that $\gamma(U)=V \cap \gamma(A(X))$. According to Theorem $3^{\prime}$ in [26], there exists an equicontinuous subset $\Phi \subseteq C(X, \mathbb{R})$ such that $\bigcap\left\{\bar{\phi}^{-1}(\mathcal{I}): \phi \in \Phi\right\} \subseteq V$, where $\mathcal{I}=(-1,1) \subset \mathbb{R}$. Because of the reflexivity of the additive group of $\mathbb{R}$, there exist an equicontinuous set $\Psi \subseteq \mathbb{R}^{\wedge}=C(\mathbb{R}, \mathbb{T})$ and a neighbourhood of the identity in $\mathbb{T}$ such that $\bigcap\left\{\psi^{-1}(W)\right.$ : $\psi \in \Psi\} \subseteq \mathcal{I}$. The subset $H=\Psi \circ \Phi$ of $C(X, \mathbb{T})$ is equcontinuous by Lemma 2. Finally,

$$
\bigcap\left\{\bar{h}^{-1}(W): h \in H\right\} \subseteq \bigcap\left\{\bar{\phi}^{-1}\left(\bigcap\left\{\psi^{-1}(W): \psi \in \Psi\right\}\right): \phi \in \Phi\right\} \subseteq V .
$$

In the sequel the subscript " $c$ " stands for the compact-open topology.

LEMma 3. The natural restriction mapping $r: C_{c}(\theta X, \mathbb{T}) \rightarrow C_{b}(X, \mathbb{T})$ is continuous.

Proof: If $C \subseteq X$ is a bounded subset and $V$ is a neighbourhood of the identity in $\mathbb{T}$, then $\operatorname{cl}_{\beta X} C \subseteq \mu X \subseteq \theta X$ and $r\left[N\left(\operatorname{cl}_{\beta X} C, V\right)\right] \subseteq N(C, V)$.

LEMma 4. If a set $H \subseteq C(X, \mathbb{T})$ is equicontinuous, then the set $\bar{H}=r^{-1}(H) \subseteq$ $C(\theta X, \mathbb{T})$ is equicontinuous.

ProOF: For every entourage of the diagonal $V$ from the (unique) uniformity of the compact group $\mathbb{T}$ we denote $\bar{H}^{-1}(V)=\left\{(x, y) \in(\theta X)^{2}:(h(x), h(y)) \in V\right.$ for all $h \in H\}$. One can assume that $V$ is closed. Since $H_{1}=\bar{H}^{-1}(V) \cap X^{2}$ belongs to the finest compatible uniform structure $\mathcal{U}_{X}$ on $X$ because of the equicontinuity of $H$, and the trace of $\mathcal{U}_{\theta X}$ on $X$ is $\mathcal{U}_{X}$, the set $\bar{H}^{-1}(V)$ is the closure of $H_{1}$ in $(\theta X)^{2}$ and therefore $\bar{H}^{-1}(V) \in \mathcal{U}_{\theta X}$.

(Here we used the following easy fact: a family of mappings $H$ from a topological space $X$ to a uniform space $Y$ is equicontinuous if and only if $B$ is equicontinuous as a family of maps from the uniform space $\left(X, \mathcal{U}_{X}\right)$ to $Y$.)

LEMMA 5. The restriction mapping $r: C(\theta X, \mathbb{T}) \rightarrow C(X, \mathbb{T})$ is onto.

Proof: Every continuous $f: X \rightarrow \mathbb{T}$ extends over $\beta X$, and $\theta X \subseteq \beta X$.

THEOREM 3. For every topological space $X$ the map $\nu: A(X) \rightarrow A(X)^{\wedge \wedge}$ is relatively open.

Proof: Let $H$ be an arbitrary equicontinuous subset of $C(X, \mathbb{T})$; the desired statement will follow immediately from Theorem 2 if we show that $H$ is precompact in $C_{b}(X, \mathbb{T})$. The set $H_{1}=r^{-1}(H)$ is equicontinuous in $C(\theta X, \mathbb{T})$ (Lemma 4). The closure $H_{2}$ of $H_{1}$ in the topology of pointwise convergence in $\mathbb{T}^{\theta X}$ is equicontinuous [16, Proposition 27], and therefore $H_{2} \subseteq C(\theta X, \mathbb{T})$. Obviously, $H_{2}$ is closed in $C_{c}(\theta X, \mathbb{T})$ and for each $x \in \theta X$ the set $\mathrm{cl}_{\mathbb{T}}\left\{h(x): h \in H_{2}\right\}$ is compact. By virtue of Ascoli's 
theorem [16, Theorem 9] $H_{2}$ is compact in $C_{c}(\theta X, \mathbb{T})$. Finally, Lemma 3 implies that $r\left(H_{2}\right)$ is compact in $C_{b}(X, \mathbb{T})$, and due to Lemma $5, H \subseteq r\left(H_{2}\right)$.

One deduces from Proposition 8 , Corollary 1 , and Theorem 3 the following result.

Corollary 2. Let $X$ be a $b_{f}$-space. Then the evaluation mapping $\nu$ is a topological isomorphism of the group $A(X)$ with a subgroup of $A(X)^{\wedge \wedge}$.

\section{SURJECTIVITY OF The EVAluation MAP AND The GROUP $\pi^{1}(X)$}

The first cohomotopy group, $\pi^{1}(X)$, of a topological space $X$ is the collection of all homotopy classes of continuous maps $X \rightarrow \mathbb{T}$, equipped with the following group operations: the product $\alpha \beta$ of two elements $\alpha, \beta \in \pi^{1}(X)$ is the class of the pointwise product of representatives from $\alpha$ and $\beta$; the element inverse to an $\alpha \in \pi^{1}(X)$ is the homotopy class of a map pointwise inverse to a representative of $\alpha[11]$.

We shall denote by $\zeta$ a group homomorphism from $C(X, \mathbb{T})$ to $\pi^{1}(X)$ assigning to every mapping its homotopy class. Let us denote by $C^{0}(X, \mathbb{T})$ the kernel of $\zeta$. We shall assume that $\pi^{1}(X)$ is topologised as a topological factor-group of $C_{b}(X, \mathbb{T})$ by its subgroup $C^{0}(X, \mathbb{T})$. Let $\xi: A(X)^{\wedge \wedge} \rightarrow C_{b}^{0}(X, \mathbb{T})^{\wedge}$ be a map dual to the embedding $C_{b}^{0}(X, \mathbb{T}) \hookrightarrow C_{b}(X, \mathbb{T})$.

PROPOSITION 9. The homomorphism $\xi \circ \nu: A(X) \rightarrow C_{b}^{0}(X, \mathbb{T})$ is mono.

Proof: Let $x \in A(X)$; we are looking for an $f \in C_{b}^{0}(X, \mathbb{T})$ such that $\bar{f}(x) \neq e_{\mathbb{T}}$. There exists a $g \in L(X)^{\prime}$ with $\alpha={ }_{\text {def }} g(\gamma(x)) \neq 0$. (Here $\gamma: A(X) \rightarrow L(X)$ is a canonical embedding.) Let $\theta: \mathbb{R} \rightarrow \mathbb{T}$ be a homomorphism given by the rule $x \mapsto$ $\exp \left(i \alpha^{-1} x\right)$. Clearly, $f=\left._{\text {def }} \theta \circ g\right|_{X}$ has the desired properties.

PROPOSITION 10. If $X$ is a $b_{f}$-space, then $\xi \circ \nu$ is a topological isomorphism of $A(X)$ onto a subgroup of $C_{b}^{0}(X, \mathbb{T})^{\wedge}$.

Proof: Since every precompact subset of $C_{b}^{0}(X, \mathbb{T})$ remains so in $C_{b}(X, \mathbb{T})$ as well, the mapping $\xi$ is continuous and therefore, in view of Corollary 1, the map $\xi \circ \nu$ is continuous. On the other hand, it follows from the proof of Theorem 2 that the topology of $A(X)$ is the topology of uniform convergence on equicontinuous subsets of $C^{0}(X, \mathbb{T})$, because the subset $H$ constructed in the process of the proof is in the latter group. It follows from the proof of Theorem 3 that every such set is precompact. Therefore, $\xi \circ \nu$ is relatively open. Proposition 9 completes the proof.

Proposition 11. The group $\pi^{1}(X)^{\wedge}$ canonically embeds in $A(X)^{\wedge \wedge}$ as a topological subgroup in such a fashion that $\nu(A(X)) \cap \pi^{1}(X)^{\wedge}=\{0\}$.

Proof: A desired canonical embedding is the map $\zeta^{\wedge}$ dual to $\zeta: C_{b}(X, \mathbb{T}) \rightarrow$ $\pi^{1}(X)$. If $x \in A(X)$, then for some $f \in C_{b}^{0}(X, \mathbb{T})$ one has $f(x) \neq 0$, while for all $\chi \in \pi^{1}(X)^{\wedge}$ one has $\chi(f)=0$. 
Corollary 3. If $A(X)$ is reflexive, then $\pi^{1}(X)^{\wedge}=(0)$.

Apparently, in the general case computation of the character group of the topologised $\pi^{1}(X)$ is not easy. But in a particular case below (Corollary 4) the result becomes quite meaningful.

Lemma 6. If $X$ is pseudocompact then $C_{b}^{0}(X, \mathbb{T})$ is open in $C_{b}(X, \mathbb{T})$.

Proof: The group $C_{b}^{0}(X, \mathbb{T})$ contains an open subset $M(X, V)$, where $V=$ $\left\{x \in \mathbb{T}:\left|x-e_{\mathbb{T}}\right|_{\mathbb{C}}<\sqrt{3}\right\}$. Indeed, if $f \in M(X, V)$, then $f$ is contractible to a constant function on $X$; the contraction at any point $x \in X$ is performed along a geodesic in $\mathbb{T}$ joining $f(x)$ and $e$.

Corollary 4. If $X$ is pseudocompact and $A(X)$ is reflexive, then $\pi^{1}(X)=$ (0).

Proof: It follows from Lemma 6 that $\pi^{1}(X)$ is a discrete group, and therefore the nontriviality of this group implies the nontriviality of $\pi^{1}(X)^{\wedge}$.

We shall see below (Section 4), that even the condition $\pi^{1}(X)=(0)$ does not ensure the reflexivity of $A(X)$.

Below we denote by $G_{d}$ a group $G$ endowed with the discrete topology, and $G_{d}^{\wedge}$ stands for $\left(G_{d}\right)^{\wedge}$.

Proposition 12. If $X$ is pseudocompact, then $A(X)^{\wedge \wedge}$ is topologically isomorphic to $C_{b}^{0}(X, \mathbb{T})^{\wedge} \oplus \pi^{1}(X)_{d}^{\wedge}$.

Proof: The group $C_{b}^{0}(X, \mathbb{T})$ is open in $C(X, \mathbb{T})$, and it is divisible (unlike, in general, $C(X, \mathbb{T}))$ - indeed, for $X$ pseudocompact, it is algebraically generated by a subset $M(X, V)$, where $V=\left\{x \in \mathbb{T}:\left|x-e_{\mathbb{T}}\right|_{\mathbb{C}}<\sqrt{3}\right\}$, and for every $f \in M(X, V)$ and each $n \in \mathbb{N}$ there is a $g \in M(X, V)$ with $n g=f$.

According to $[10,6.22 . \mathrm{b}]$, the group $C_{b}(X, \mathbb{T})$ is topologically isomorphic to $C_{b}^{0}(X, \mathbb{T}) \oplus \pi^{1}(X)$. Theorem 13 in [16] finishes the proof. (Clearly, it remains valid for all - not just locally compact - topological groups, and also in the case where the character group is endowed with our "precompact-open" topology.)

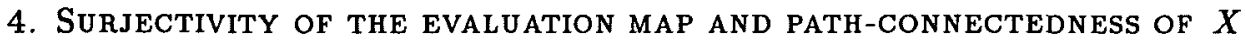

Let $X$ be a topological space. Denote by $\theta$ a map of the linear space $C(X, \mathbb{R})$ to the group $C(X, \mathbb{T})$, given by the rule $\theta(f)(x)=\exp (2 \pi i f(x))$. The image of $C(X, \mathbb{R})$ under $\theta$ is contained in $C^{0}(X, \mathbb{T})$ and $\theta$ is an additive group homomorphism.

If $x_{0} \in X$, denote

$$
\begin{gathered}
C\left(X, x_{0}, \mathbb{R}\right)=\left\{f \in C(X, \mathbb{R}): f\left(x_{0}\right)=0\right\}, C\left(X, x_{0}, \mathbb{T}\right)=\left\{f \in C(X, \mathbb{T}): f\left(x_{0}\right)=e_{\mathbb{T}}\right\}, \\
C^{0}\left(X, x_{0}, \mathbb{T}\right)=\left\{f \in C^{0}(X, \mathbb{T}): f\left(x_{0}\right)=e_{\mathbb{T}}\right\} \equiv C^{0}(X, \mathbb{T}) \cap C\left(X, x_{0}, \mathbb{T}\right) .
\end{gathered}
$$


Obviously, $\theta$ maps $C\left(X, x_{0}, \mathbb{R}\right)$ to $C^{0}\left(X, x_{0}, \mathbb{T}\right)$. Denote by $\theta_{0}$ the restriction of $\theta$ to $C\left(X, x_{0}, \mathbb{R}\right)$.

Proposition 13. Let $X$ be a path-connected space and let $x_{0} \in X$. Then the map $\theta_{0}: C\left(X, x_{0}, \mathbb{R}\right) \rightarrow C^{0}\left(X, x_{0}, \mathbb{T}\right)$ is an algebraic isomorphism. If in addition $X$ is a pseudocompact space, then $\theta_{0}$ is a topological isomorphism between $C_{b}\left(X, x_{0}, \mathbb{R}\right)$ and $C_{b}^{0}\left(X, x_{0}, \mathbb{T}\right)$.

PROOF: The first statement follows from the covering mapping theorem $[8,5.6 .19$, 11]. The continuity of $\theta_{0}$ is obvious. Now let $0<\varepsilon<\sqrt{3}$. Set $V_{\varepsilon}=\{\exp (i x)$ : $|x|<\varepsilon\} \subseteq \mathbb{T}$. We shall show now that $\theta_{0}^{-1}\left(N\left(X, V_{\varepsilon}\right)\right) \subseteq N(X,(-\varepsilon, \varepsilon)$ ) (the notation is obvious). If $f \in N\left(X, V_{\varepsilon}\right)$, then $\theta_{0}^{-1}(f)\left(x_{0}\right)=0$ and, by virtue of the path-connectedness of $X, \theta_{0}^{-1}(f)(X)$ is in the same path component of $\theta_{0}^{-1}\left(V_{\varepsilon}\right)=$ $\bigcap\{(n-\varepsilon, n+\varepsilon): n \in \mathbb{N}\}$ as 0 is, that is, in $(-\varepsilon, \varepsilon)$.

Lemma 7. For every element $x_{0} \in X$ the groups $C_{b}^{0}(X, \mathbb{T})$ and $C_{b}^{0}\left(X, x_{0}, \mathbb{T}\right) \oplus \mathbb{T}$ are topologically isomorphic under the mapping $f \mapsto\left(f \cdot f\left(x_{0}\right)^{-1}, f\left(x_{0}\right)\right)$.

For a compact space $X$ denote by $M(X)$ the Banach space of all regular Borel measures on $X$, that is, the strong dual to the space $C_{c}(X, \mathbb{R})$.

THEOREM 4. If $\nu: A(X) \rightarrow A(X)^{\wedge \wedge}$ is onto then $X$ is totally path-disconnected, that is, every path-component of $X$ is a singleton.

ProOF: Assume that $\nu$ is onto and at the same time there exists a path-component of $X$ containing more than one point. Denote by $C$ a non-trivial image of the closed interval $[0,1]$ in $X$. Let $r$ stand for the restriction mapping from $C_{b}(X, \mathbb{T})$ to $C_{b}(C, \mathbb{T})$. Since every compact subset of a completely regular space is $C^{*}$-embedded, $r$ is onto. By virtue of the continuity of $r$, the dual map $r^{\wedge}: C_{b}(X, \mathbb{T}) \rightarrow C_{b}(X, T)^{\wedge} \equiv A(X)^{\wedge \wedge}$ is defined correctly. Since $r$ is onto, $r^{\wedge}$ is a monomorphism.

Let $m \in M(C), m: C_{b}(C, \mathbb{R}) \rightarrow \mathbb{R}$. Fix $c_{0} \in C$. The mapping $\chi_{m}^{\prime}: C_{b}^{0}\left(C, c_{0}, \mathbb{T}\right) \rightarrow$ $\mathbb{T}$, given by the rule

$$
f \mapsto \exp \left[2 \pi i m\left(\theta_{0}^{-1}(f)\right)\right],
$$

is a continuous homomorphism according to Proposition 13. Proposition 12 and Lemma 7 imply that $\chi_{m}^{\prime}$ extends to a continuous homomorphism $\chi_{m}: C_{b}(C, \mathbb{T}) \rightarrow \mathbb{T}$. Since $\chi_{m} \in A(X)^{\wedge \wedge} \equiv \nu(A(X))$ and $\nu^{-1} \chi_{m} \in A(X)$, one can find a finite subset $X^{\prime}=$ $\left\{x_{1}, \ldots, x_{m}\right\} \subseteq X$ such that for every $f \in C(C, \mathbb{T})$ the property $f\left(X^{\prime}\right) \subseteq\left\{e_{\mathbb{T}}\right\}$ implies $\chi_{m}(f)=e_{\mathbb{T}}$.

If now $\phi \in C\left(X, x_{0}, \mathbb{R}\right)$ and in addition $\phi\left(X^{\prime}\right) \subseteq\{0\}$, then the function $\psi: x \mapsto$ $\exp [2 \pi i \phi(x)]$ belongs to $C^{0}\left(X, x_{0}, \mathbb{T}\right)$ and $\psi\left(X^{\prime}\right) \subseteq\left\{e_{\mathbb{T}}\right\}$, so that $\chi_{m}(\psi)=e_{\mathbb{T}}$. At the same time, $\chi_{m}(\psi)=\exp \left[2 \pi i m\left(\theta_{0}^{-1}(\psi)\right)\right]=\exp [2 \pi i m(\phi)]$, and therefore $m(\phi) \in \mathbb{Z}$. The same applies to the function $\phi_{1}=\pi \phi$, and we conclude: $m(\phi) \in \mathbb{Z} \cap \pi^{-1} \mathbb{Z}=\{0\}$. 
This means that the support of $m$ is finite. We have arrived at a contradiction, because $C$ is an infinite compact set and $m$ is an arbitrary measure on it.

In [18] the statement of Theorem 4 was obtained in the case where $X$ is a $k_{\omega}$-space.

\section{A Class OF REFLEXIVE GROUPS $A(X)$}

For a topological space $X$ we denote

$$
E(X)=\bigcup\left\{\operatorname{cl}_{\beta X} B: B \in \mathcal{B}\right\},
$$

where $\mathcal{B}$ is the family of all bounded subsets of $X$. Clearly, $X \subseteq E(X) \subseteq \mu X$. However, $E(X)$ need not coincide with $\mu(X)$ [4], though if $X=E(X)$ then $X$ is a $\mu$-space.

If $\gamma$ is a disjoint cover of $X$, we call a mapping $f: X \rightarrow Y \gamma$-labelled if the restriction of $f$ to each member of $\gamma$ is a constant map.

Denote by $\Gamma_{X}$ the totality of all finite disjoint open covers of the space $X$.

Lemma 8. Let $\operatorname{dim} X=0$. The subset of all mappings which are $\gamma$-labelled for some $\gamma \in \Gamma_{X}$ (depending on a mapping) is uniformly dense in $C(X, \mathbb{T})$.

Proof: Let $f \in C\left(X, \mathbb{T}\right.$ ) and let $V$ be a neighbourhood of $e_{\mathbb{T}}$ in $\mathbb{T}$ (we assume that $\left.|V|_{\mathbb{C}} \subseteq(-\sqrt{3}, \sqrt{3})\right)$. Let $W$ be open in $\mathbb{T}$ and such that $W \cdot W^{-1} \subseteq V$. Choose a finite set $A=\left\{a_{1}, \ldots, a_{n}\right\} \subset \mathbb{T}$ with $A \cdot W=\mathbb{T}$. The family $\left\{f^{-1}\left(a_{i} W\right): i=\right.$ $1, \ldots, n\}$ forms a functionally open cover of $X$; refine it to a finite disjoint open cover $\gamma$. For each $U \in \gamma$ fix an $x_{U} \in U$ and set $\phi(U)=\left\{f\left(x_{U}\right)\right\}$. The resulting map $\phi$ is $\gamma$-labelled and for every $x \in X$ one has $\phi(x) f(x)^{-1}=f\left(x_{U}\right) f(x)^{-1}$, if $x \in U \in$ $\gamma$. For a suitable $i=1,2, \ldots, n$ one has $U \subseteq f^{-1}\left(a_{i} W\right)$, therefore $f\left(x_{U}\right) f(x)^{-1} \in$ $f(U) f(U)^{-1} \subseteq a_{i} W\left(a_{i} W\right)^{-1}=W W^{-1} \subseteq V$.

Theorem 5. Let $X$ be a $\mu$-space and $\operatorname{let} \operatorname{dim} X=0$. Then $\nu: A(X) \rightarrow A(X)^{\wedge \wedge}$ is onto.

Proof: Let $\chi \in A(X)^{\wedge \wedge}, \chi: C_{b}(X, \mathbb{T}) \rightarrow \mathbb{T}$. We shall define an integer-valued function $i_{x}$ on the set $\mathcal{V}$ of all open-and-closed subsets of $X$ as follows. Let $V \in \mathcal{V}$. Denote by $G_{V}$ the subgroup of $C(X, \mathbb{T})$ formed by all mappings $f$ such that $f(V)$ is a singleton and $f(X \backslash V) \subseteq\left\{e_{\mathbb{T}}\right\}$. Clearly, $G_{V}$ is topologically isomorphic to $\mathbb{T}$ under the mapping $f \mapsto f(V)$. Set $i_{\chi}(V)$ to be equal to $k$, if the restriction of $\chi$ to $G_{V}$ is of the form $\exp (2 \pi i x) \mapsto \exp (2 \pi k i x)$ under the above identification $G_{V} \cong \mathbb{T}$; in other words, $i_{\chi}(V)$ is simply the degree of the mapping $\left.\chi\right|_{G_{V}}: G_{V} \rightarrow \mathbb{T}$.

The set $\Gamma_{X}$ is naturally directed: $\gamma \prec \gamma^{\prime}$ if $\gamma$ is a refinement of $\gamma^{\prime}$. Any family of sets of the form $\left\{V_{\gamma}: \gamma \in \Gamma_{X}\right\}$, where $V_{\gamma} \in \gamma$ and $V_{\gamma} \subseteq V_{\gamma^{\prime}}$ whenever $\gamma \prec \gamma^{\prime}$, forms a basis for a Cauchy filter with respect to the finest totally bounded compatible 
uniformity on $X$, which we denote by $\mathcal{C}^{*}(X)$. Since the completion of the uniform space $\left(X, \mathcal{C}^{*}(X)\right)$ is $\beta X[5,8.3 .18]$, each family of the form $\left\{V_{\gamma}: \gamma \in \Gamma_{X}\right\}$ with the above properties converges to some element of $\beta X$. Moreover, each element of $\beta X$ can be obtained in this way.

Denote by $S_{x}$ the collection of all $x \in \beta X$ which are limits of prefilters of the form $\left\{V_{\gamma}: \gamma \in \Gamma_{X}\right\}$, where $V_{\gamma} \in \gamma$ and $V_{\gamma} \subseteq V_{\gamma^{\prime}}$ if $\gamma \prec \gamma^{\prime}$, with the additional property that for every neighbourhood $U \ni x$ there is a $\gamma \in \Gamma_{X}$ such that $V_{\gamma} \subseteq U$ and $i_{\chi}\left(V_{\gamma}\right) \neq 0$.

Claim 1. $S_{\chi}$ is a finite set.

$\triangleleft$ Assuming the contrary, one can easily construct by induction a countable relatively discrete (that is, discrete in itself) set $\left\{s_{n}: n \in \mathbb{N}\right\} \subseteq S_{\chi}$, and a disjoint family $\left\{V_{n}: \in \mathbb{N}\right\}$ of open-and-closed subsets of $X$ such that for all $n \in \mathbb{N}$ one has $s_{n} \in \operatorname{cl}_{\beta} X V_{n}$ and $i_{\chi}\left(V_{n}\right)=i_{n} \neq 0$. For $n \in \mathbb{N}$ define the function $f_{n} \in G_{V_{n}}$ by letting $f_{n}(x)=e_{\mathbb{T}}$ for $x \in X \backslash V_{n}$ and $f_{n}(x)=\exp \left(2 \pi i n^{-1} i_{n}^{-1}\right)$ for $x \in V_{n}$. Since for every neighbourhood $W$ of the identity in $\mathbb{T}$ one has $f_{n}(X) \subseteq W$ for all $n$ starting from some natural number, the function $f=\sum_{n \in \mathbf{N}} f_{n}$ is continuous on $X$. Furthermore, $f$ is a uniform limit of the sequence of functions $\left(\sum_{n=1}^{m} f_{n}\right)$ as $m \rightarrow \infty$, and therefore one should have $\chi(f)=\prod_{n \in \mathbb{N}} \chi\left(f_{n}\right)=\prod_{n \in \mathbf{N}} \exp \left(2 \pi i n^{-1}\right)$. However, the latter infinite product diverges in T. $\triangleright$

Since every function from $C(X, \mathbb{T})$ extends in a unique way to a continuous $\mathbb{T}$ valued mapping on $\beta X$, the group $C_{b}(X, \mathbb{T})$ can be canonically identified with the group $C(\beta X, \mathbb{T})$, equipped with the topology of uniform convergence on bounded subsets of $X$. Every $f \in C(X, \mathbb{T})$ can be thought of as a mapping $\beta X \rightarrow \mathbb{T}$.

Claim 2. If $f \in C(X, \mathbb{T})$, then $\chi(f)=\prod_{\bullet \in S} f(s)^{n_{s}}$ for an appropriate collection $n_{\bullet} \in \mathbb{Z}$, $s \in S$.

$\triangleleft$ If a $\gamma$-labelled function $f$ equals $e_{\mathbb{T}}$ on $S$, then it assumes the same value on some neighbourhood $U$ of $S$, which can be assumed to be open-and-closed. By the definition of $S_{\chi}$, for each $x \in \beta X \backslash U$ there exist $\gamma \in \Gamma_{X}$ and $V_{x} \in \gamma$ such that the value of $i_{x}$ on every open-and-closed subset of $V_{x}$ is 0 . Refine the cover $\left\{V_{x}: x \in \beta X \backslash U\right\}$ to a finite disjoint open-and-closed subcover $\gamma_{1}$ of $\beta X \backslash U$. (It is possible to do this because $\beta X \backslash U$ is a strongly zero-dimensional compact space.) For every $V \in \gamma_{1}$, define a function $f_{V}$ by

$$
f_{v}(x)= \begin{cases}f(x), & \text { if } x \in V \\ e_{\mathbb{T}}, & \text { otherwise }\end{cases}
$$

Clearly, for every $V \in \gamma_{1}$, one has $\chi\left(f_{v}\right)=e_{\mathbb{T}}$, because $i_{\chi}(V)=0$. Since $\left.f\right|_{U} \equiv e_{\mathbb{T}}$, one 
must have $f=\prod_{V \in \gamma_{1}} f_{V}$ (finitely many factors) and therefore $\chi(f)=\prod_{V \in \gamma_{1}} \chi\left(f_{V}\right)=e_{\mathbb{T}}$.

A straightforward consequence is that $\chi(f)=\chi(g)$ whenever $f$ and $g$ are two $\gamma$-labelled functions coinciding on $S$.

Now let $g$ be an arbitrary $\gamma$-labelled function on $S$. Fix disjoint open subsets of $\beta X, U_{s}, s \in S$, such that $s \in U_{s}$ for all $s$ and the function $g$ is constant on each $U_{s}$. Set $n_{s}=i_{\chi}\left(U_{s}\right)$. Define a function $f: \beta X \rightarrow \mathbb{T}$ by letting $f \equiv 0$ outside $\bigcup\left\{U_{s}: s \in S\right\}$ and $f\left(U_{s}\right)=\left\{g(s)^{n_{s}}\right\}$. Obviously, $\chi(f)=\prod_{s \in S} f(s)^{n_{s}}$ and, by the preceding paragraph, $\chi(g)=\chi(f)=\prod_{s \in S} g(s)^{n_{\iota}}$. Lemma 8 finishes the proof of Claim 2: there is a net $\left(f_{\alpha}\right)$ of $\gamma_{\alpha}$-labelled functions uniformly converges to a function $f \in C(X, \mathbb{T})$, and one can assume that $\left.f_{\alpha}\right|_{S} \equiv e_{\mathbb{T}}$ for all $\alpha$; therefore, $\chi(f)=\lim _{\alpha} \chi\left(f_{\alpha}\right)=\prod_{s \in S} f(s)^{n_{s}}$. D

Claim 3. $S \subseteq E(X)$.

$\triangleleft$ Let $W$ be a neighbourhood of the identity in $\mathbb{T}$. There exist a bounded subset $B \subseteq X$ and a neighbourhood $U$ of the identity in $\mathbb{T}$ such that $\chi(f) \in W$ whenever $f(B) \subseteq U$. If for some $x \in S$ one has $x \notin \operatorname{cl}_{\beta X} B$, then there exists a continuous function $f: \beta X \rightarrow \mathbb{T}$ with the properties $f(B)=\left\{e_{\mathbb{T}}\right\}, f(S \backslash\{x\}) \subseteq\left\{e_{\mathbb{T}}\right\}$, and $f(x)^{n_{x}} \notin W$. Now $\chi(f)=\prod_{\theta \in S} f(x)^{n_{s}}=f(x)^{n_{x}} \notin W$, a contradiction, since $f(B)=\left\{e_{\mathbb{T}}\right\} \subseteq U$.

Our Theorem is proved. Indeed, since $X$ is a $\mu$-space, one has $E(X)=X$, therefore $S \subseteq X$, and $\chi=\nu\left(\sum_{x \in S} n_{x} x\right)$, where $\sum_{x \in S} n_{x} x \in A(X), S$ being finite.

Combining the statements of Proposition 9 and Theorems 1, 3 and 5, we obtain our main result.

TheOREM 6. Let $X$ be a $\mu$-space and $k_{f}$-space, and let $\operatorname{dim} X=0$. Then $A(X)$ is a reflexive topological group.

Corollary 5. Let $X$ be a paracompact $k$-space and let $\operatorname{dim} X=0$. Then $A(X)$ is a reflexive topological group.

COROLlaRY 6 . Let $X$ be a zero-dimensional compact space or a strongly zerodimensional metrisable space. Then $A(X)$ is a reflexive topological group.

In particular, the free Abelian topological groups $A(\mathbb{Q})$ and $A\left(\mathbb{Z}^{\mathbb{N}_{0}}\right)$ on both rational and irrational numbers provide examples of reflexive groups of a type completely unknown before.

\section{CONCLUDING REMARKS}

1. Does any known noncommutative analogue of Pontryagin-van Kampen duality (such as Tannaka-Krein duality) work for Markov free topological groups? 
2. Answering a question by Noble [19], Nickolas [18] had shown that a complete topological $k$-group, whose points are separated by continuous characters, need not be reflexive: his counter-example was $A[0,1]$.

However, a non-discrete free Abelian topological group is never Čech-complete [1]. Is every complete and Čch-complete topological group, whose points are separated by continuous characters, reflexive?

\section{REFERENCES}

[1] A.V. Arhangel'skiř, 'Relations among invariants of topological groups and their subspaces', Russian Math. Surveys 35 (1980), 1-23.

[2] A.V. Arhangel'skií, 'Linear homeomorphisms of function spaces', Soviet Math. Dokl. 25 (1982), 852-855.

[3] W. Banaczcyk, Additive subgroups of topological vector spaces, Lecture Notes in Mathematics 1466 (Springer-Verlag, Berlin, Heidelberg, New York, 1991).

[4] J.L. Blasco, 'On $\mu$-spaces and $\boldsymbol{k}_{R}$-spaces', Proc. Amer. Math. Soc. 67 (1977), 179-186.

[5] R. Engelking, General topology (PWN, Warczawa, 1977).

[6] J. Flood, Free topological vector spaces, (Ph.D. thesis) (Australian National University, Canberra, 1975).

[7] J. Flood, Free locally convex spaces, Dissert. Math. CCXXI (PWN, Warsczawa, 1984).

[8] D.B. Fuks and V.A. Rokhlin, Beginner's Course in Topology: Geometric Chapters, (translated from the Russian by A. Iacob) (Springer-Verlag, Berlin, Heidelberg, New York, 1984).

[9] B.R. Gelbaum, 'Free topological groups', Proc. Amer. Math. Soc. 12 (1961), 737-743.

[10] E. Hewitt and K.A. Ross, Abstract harmonic analysis. I, (second edition) (Springer-Verlag, Berlin, Heidelberg, New York, 1979).

[11] S.-T. Hu, Homotopy theory, Pure and Applied Mathematics, 8 (Academic Press, New York, London, 1959).

[12] S. Kaplan, 'Extensions of the Pontryagin duality, I. Infinite products', Duke Math. J. 15 (1948), 649-658.

[13] A.A. Kirillov and A.D. Gvishiani, Theorems and problems in functional analysis, (translated from Russian by Harold H. McFaden) (Springer-Verlag, Berlin, Heidelberg, New York, 1982).

[14] A.A. Markov, 'Three papers on topological groups', Amer. Math. Soc. Transl. 30 (1950), $120 \mathrm{pp}$.

[15] E. Martin-Peinador, 'A reflexive admissible topological group must be locally compact', Proc. Amer. Math. Soc. (to appear).

[16] S.A. Morris, Pontryagin duality and the structure of locally compact Abelian groups (Cambridge University Press, Cambridge, London, New York, Melbourne, 1977).

[17] S.A. Morris, 'Free Abelian topological groups', in Categorical Topology, Proc. Conference Toledo, Ohio, 1983 (Heldermann-Verlag, 1984), pp. 375-391. 
[18] P. Nickolas, 'Reflexivity of topological groups', Proc. Amer. Math. Soc. 65 (1977), 137-141.

[19] N. Noble, 'k-Groups and duality', Trans. Amer. Math. Soc. 151 (1970), 551-561.

[20] E. Nummela, 'The completion of a topological group', Bull. Austral. Math. Soc. 21 (1980), 407-417.

[21] V. Pestov, 'Some properties of free topological groups', Moscow Univ. Math. Bull. 37 (1982), 46-49.

[22] V. Pestov, 'Free topological Abelian groups and the Pontryagin duality', Moscow Univ. Math. Bull. 41 (1986), 1-4.

[23] V. Pestov, 'Universal arrows to forgetful functors from categories of topological algebra', Bull. Austral. Math. Soc. 48 (1993), 209-249.

[24] D.A. Raikov, 'Harmonic analysis on commutative groups with Haar measure and the theory of characters', (in Russian), Trudy Mat. Inst. Steklov 14 (1945), 1-86.

[25] D.A. Raǐkov, 'On the completion of topological groups', (in Russian), Izv. Akad. Nauk SSSR. Ser. Mat. 10 (1946), 513-528.

[26] D.A. Ray̌kov, 'Free locally convex spaces for uniform spaces', (in Russian), Mat. Sb. (N.S.) 63 (1964), 582-590.

[27] D. Remus and F.J. Trigos-Arrieta, 'Abelian groups which satisfy Pontryagin duality need not respect compactness', Proc. Amer. Math. Soc. 117 (1993), 1195-1200.

[28] M. Smith, 'The Pontryagin duality theorem in linear spaces', Ann. of Math. 56 (1952), 248-253.

[29] M. G. Tkachenko, 'On completeness of free Abelian topological groups', Soviet Math. Dokl. 27 (1983), 341-345.

[30] V.V. Uspenskiri, 'On the topology of free locally convex space', Sov. Math. Dokl. 27 (1983), 781-785.

[31] R.F. Wheeler, 'Weak and pointwise compactness in the space of bounded continuous functions', Trans. Amer. Math. Soc. 266 (1981), 515-530.

Department of Mathematics

Victoria University of Wellington

Wellington

New Zealand

e-mail: vladimir.pestov@vuw.ac.nz 